\title{
Serratia marcescens Sepsis in a Child with Deep Venous Thrombosis - A Case Report
}

\author{
Iulia Armean ${ }^{1}$, Carmen Duicu2 ${ }^{*}$, Cornel Aldea ${ }^{3}$, Lorena Melit ${ }^{1}$ \\ 1 Pediatric Clinic No 1, County Emergency Clinical Hospital, Tirgu Mures, Romania \\ $21^{\text {st }}$ Department of Pediatrics, University of Medicine and Pharmacy of Tirgu Mureș, Romania \\ $32^{\text {nd }}$ Pediatric Clinic, Clinical Emergency Hospital for Children, Cluj-Napoca, Romania
}

\begin{abstract}
Introduction: Venous thromboembolism is a rare condition in paediatrics that included both deep venous thrombosis and pulmonary embolism. Serratia marcescens is a gram-negative bacterium that belongs to the Enterobacteriaceae family and tends to affect immunocompromised hosts.

Case report: We report the case of an 11-year-old boy, admitted in the Pediatric Clinic I from Emergency County Hospital Tîrgu Mureș, Romania with intense pain, swelling, cyanosis and claudication of the left foot. His personal history revealed a recent appendectomy. A close family was reported to have had a deep venous thrombosis. The laboratory tests, performed on the day of admission, revealed increased inflammatory biomarkers and D-dimer. Coagulation tests gave a low activated partial thromboplastin time (APTT). Doppler venous ultrasound and CT-exam established a diagnosis of deep venous thrombosis. Anticoagulant therapy was initiated, but on the tenth day of admission, the patient developed signs and symptoms of sepsis, and the blood culture revealed Serratia marcescens. After antibiotic and anticoagulant therapy, the patient progressed favourably. The patient was a carrier of the heterozygous form of Factor V Leiden.
\end{abstract}

Conclusions: The association between deep venous thrombosis and Serratia marcescens sepsis can compromise a condition in pediatric patients.

Keywors: Serratia marcescens, sepsis, deep venous thrombosis, child

Received: 29 October 2017 / Accepted: 29 January 2018

\section{INTRODUCTION}

Venous thromboembolism (VTE) remains a rare condition in children that comprises both deep venous thrombosis (DVT) and pulmonary embolism (PE), despite the reports from to the recent studies, which state that its incidence is increasing in pediatric patients ( 1 $3)$. In the USA, this condition has a prevalence of approximately 100:100,000 individuals every year, with the incidence increasing with age. Thus, it seems that in children under the age of 15 years, the incidence is of approximately 5:100,000 cases, whereas in adult above the age of 80 years, this condition can reach an incidence of 500:100,000 cases (4). Even though a precise estimation of VTE in children cannot be established because there are only a few reported prospective stud- ies, these studies underlined an annual incidence of pediatric VTE between 0.07-0.14:10,000 children and 5.3:10,000 admissions to pediatric hospitals (5). VTE is a multifactorial condition, and most of the children that develop this disorder, present multiple risk factors (1). The gold standard for the diagnosis of DVT is angio-CT. Recently, venous compression ultrasound is the initial diagnostic tools used in these cases due to the advantages related to the costs, availability and safety profile $(4,6)$. Current treatment of VTE includes unfractionated heparin, low molecular weight heparin, and warfarin, but fondaparinux and direct thrombin inhibitors can also be used (7).

Perioperative anticoagulation is not commonly done in children without known prothrombotic conditions due to the haemorrhage risk and the very low VTE in-

\footnotetext{
* Correspondence to: Carmen Duicu, 1st Department of Pediatrics, University of Medicine and Pharmacy Tirgu Mures, 38 Gh. Marinescu St., 540139 , Tirgu Mures, Romania. 
cidence in this age group. This may cause a delay in the diagnosis and appropriate anticoagulant therapy resulting in undesirable developments.

Serratia marcescens is a gram-negative bacterium of the Enterobacteriaceae family and tends to affect immunocompromised hosts. Risk factors for Serratia marcescens infection include neonatal period, surgical interventions, mechanical ventilation, hospitalised patients, and patients with central venous catheters (8). Bloodstream infections are one of the most common causes of morbidity and mortality in newborns and children (9). Bacteremia in pediatric age-groups can give rise to self-limiting infections, life-threatening sepsis, multiple organ failures, or even disseminated intravascular coagulation (10).

Sepsis represents a systemic inflammatory response that includes two or more of the following criteria: tachypnea; tachycardia, hyperthermia or hypothermia and leukocytosis or leukopenia (11).

Hemostasis imbalance is often associated with sepsis. The laboratory modifications vary from minimal alterations such as low platelet count, leucocytosis or hyperbilirubinemia to general microvascular thrombosis, leading to organ dysfunction.

As a result of the systemic inflammatory activation, there is a reciprocal correlation between sepsis and the coagulation pathway (12).

This case aims to report a rare episode of Serratia marcescens infection in a child with DVT, which lead to clinical sepsis.

\section{- CASE REPORT}

\section{Medical history and presenting concerns}

The case describes an 11-year-old boy, admitted to the Pediatrics Clinic with intense pain, swelling, cyanosis and claudication of the left foot. These symptoms had first occurred approximately two days before the admission. His personal history revealed recent surgery for appendicitis. His father had been diagnosed with deep venous thrombosis.

\section{Clinical findings}

The clinical examination, on admission, showed an altered general status, ringed face, pallor, cyanosis, swelling and mottled skin of the left thigh and leg, and also severe pain on the mobilization of the left foot. The patient weighted 43 kilograms. The diameter of the left leg was $45 \mathrm{~cm}$, right leg $43 \mathrm{~cm}$, left ankle $29 \mathrm{~cm}$, right ankle $28 \mathrm{~cm}$. The physical exam revealed no other abnormalities.

\section{Diagnostic focus and assessment}

The laboratory tests performed on the day of admission revealed increased inflammatory biomarkers with C-reactive protein (CRP) $74.1 \mathrm{mg} / \mathrm{L}$, and D-dimer $>5$ $\mu \mathrm{g} / \mathrm{ml}$. Coagulation tests showed a low activated partial thromboplastin time (APTT) of 18,9 seconds. The chest X-ray and cardiology exam revealed no relevant pathology. Doppler ultrasound showed an enlarged and uncompressed left commune iliac vein, extern iliac vein, common femoral vein, superficial femoral vein, deep femoral vein, with hyperechoic aspect inside the lumen (Fig 1). The angio-CT exam confirmed the ultrasound findings, establishing the diagnosis of deep venous thrombosis affecting the same veins mentioned previously (Fig 2). Laboratory tests were performed to rule out a possible thrombophilia, and the results underlined that antithrombin III, von Willebrand factor, protein $\mathrm{C}$, protein $\mathrm{S}$ and homocysteine were all within the normal ranges. Genetic tests indicated a mutation of factor V Leiden (A506G). There was no abnormality in prothrombin G20210A ( factor II mutation). Lupus anticoagulant, anti-cardiolipin antibodies and beta2glycoprotein I were all within normal limits, excluding a diagnosis of antiphospholipid syndrome.

\section{Therapeutic focus and assessment}

Efficient anticoagulant therapy with low molecular weight heparin was started. The patient presented a slowly favourable evolution. On the $10^{\text {th }}$ day after hospitalisation, his general status worsened, and he developed a fever, shaking chills, vomiting, low blood pressure and prolonged capillary refill time. Laboratory tests revealed leukopenia $(2760 / \mu \mathrm{L})$ and anaemia (haemoglobin $10.5 \mathrm{~g} / \mathrm{dl}$, hematocrit $31.7 \%$, erythrocytes $3850000 / \mu \mathrm{L}$ ), with an increased CRP level $(153.47 \mathrm{mg} / \mathrm{L})$. Empiric double-regimen antibiotic therapy, Ceftazidime $2 \mathrm{~g} 2$ times a day and Ampicillin 1,5 g 4 times a day for 5 days, was initiated until the result of the blood culture were obtained. Blood culture revealed Serratia marcescens. According to the antibiogram, the bacterium was sensitive only to piperacillin/tazobactam, ertapenem, imipenem, meropenem, amikacin, and tigecycline. The empirical antibiotic therapy was replaced by Amikacin, 320 mg 2 times a day for ten days, and this resulted in a favorable improvement. The patient was discharged in 
good general status. It was recommended he received anticoagulation therapy and prevention therapy with compressive elastic hosiery.

\section{DISCUSSIONS}

VTE is a severe condition in children of major clinical significance due to its increased morbidity and mortality rates. Regarding pediatric patients, infants under the age of 1 year are the most commonly affected age group, followed by adolescents $(5,13,14)$. The present report concerns an 11 years old who developed DVT and is similar to a previously published case report (6). The annual incidence of VTE varies depending on the geographical area, being much lower in Asiatic people than in Caucasian populations (16); our patient belongs in the latter type of population. The genetic risk for thrombosis is well-documented, but there are also other factors that concur to the development of this condition, such as surgical intervention, trauma, malignant diseases, trauma, central venous catheters, veins abnormalities or chronic inflammatory conditions $(1,6,14)$. The patient had a genetic risk factor as his father had been diagnosed with DVT at a young age. Additionally, he had external risk factors, having had an appendectomy two weeks before the diagnosis of DVT and subsequent bed rest plus infection. In comparison to adults, where up to $50 \%$ of the first VTE episodes have an idiopathic underlying condition, VTE in children has a multifactorial pathogenesis, and almost $90 \%$ of these cases have at least two risk factors for thrombosis at the time of the event (16-18).

Specific inherited defects, like the factor V Leiden mutation, the prothrombin gene mutation, and the protein $\mathrm{C}$, protein $\mathrm{S}$, and antithrombin deficiencies are well-known to be associated with VTE development $(19,20)$. In our patient, the heterozygous state for Factor V Leiden increases the chance of developing thrombosis by four to eight times. The recurrence rate of VTE remains high, despite the administration of anticoagulant therapy, being of approximately $7 \%$ at six months, with a mortality rate of $6 \%$ for DVT and $12 \%$ for PE within one month (16). Fortunately, after showing slow progress, in the current patient, complete permeabilisation was achieved after twelve months of treatment, and he was, at the time of the writing of this case report, recurrence-free.

Because of thrombophilia, short prophylactic treatment is necessary during periods of high risk of thrombosis, such as dehydration, surgery, etc. It is essential to advise the family that external prothrombotic risk factors such as sedentary lifestyle, dehydration, smoking and overweight/obesity should be avoided.

The incidence of Serratia marcescens, an opportunistic organism, is high among immunocompromised and debilitated hosts (7). Our patient is considered to be a debilitated host due to the recent appendectomy and prolonged hospitalization, firstly due to the surgical intervention and afterwards to the DVT episode. Mortality related to Serratia marcescens bacteremia is reported to be between $10-20 \%(21,22)$. A wide range of incidence values for bacteremia in pediatric patients

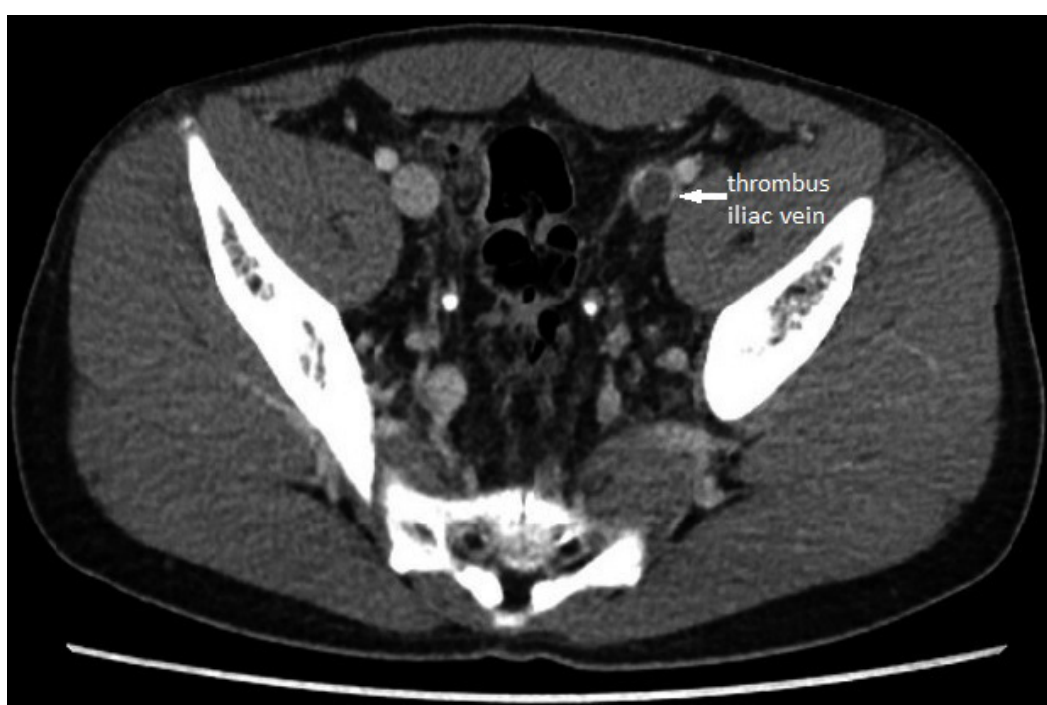

Fig1. CT scan - thrombus in the left common iliac vein (white arrow)

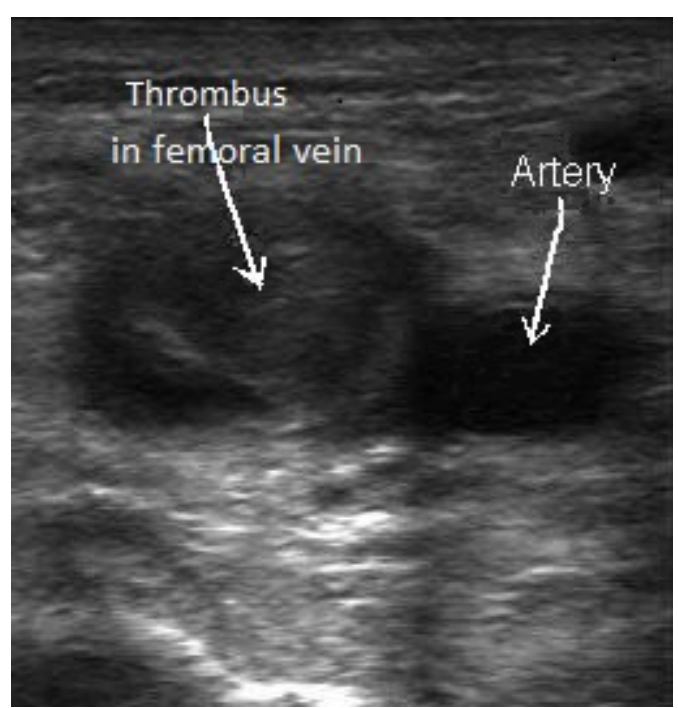

Fig 2. Transverse Ultrasound aspect: echogenic thrombus in the left common femoral vein (LCFV), non-compressible LCFV 
presents have been reported in the literature and physicians should be aware of the importance of identifying the bloodstream pathogens to improve the patient's outcome by the administration of appropriate antibiotics. Identification from blood cultures of the causative pathogen is a significant challenge in the management of sepsis. Therefore, empirical antibiotic therapy represents a key component in this management, and it should be based on the assessment of the most likely pathogen depending on the age, gender, host's risk factors, but also antibiotic susceptibility patterns (9).

A double-regimen empirical antibiotic therapy was employed in the present case, which was adjusted according to blood culture results and the subsequent antibiogram. Nevertheless, it is evident that the administration of a targeted antibiotic therapy reduces the mortality rates related to bloodstream infections (9).

\section{- CONCLUSIONS}

Even though VTE is a rare condition in paediatrics, it is known that both congenital and acquired conditions can contribute to the development of thrombosis. Serratia marcescens tends to affect immunocompromised hosts such as post-surgical ones and is a very rare bacteria found in pediatric pathology. Serratia marcescens sepsis was not initially considered to be related to the DVT, due to their relatively rare association, and resulted in delayed appropriate therapy and the resolution of the patient's condition.

\section{CONFLICT OF INTEREST}

None to declare.

\section{REFERENCES}

1. Choi HS, Choi CW, Kim HM, Park HW. Venous thromboembolism in pediatric patients: a single institution experience in Korea. Blood Res. 2016;51:164-170.

2. Chalmers E, Ganesen V, Liesner R, et al. Guideline on the investigation, management and prevention of venous thrombosis in children. Br J Haematol. 2011;154:196-207.

3. Nowak-Göttl $U$, Janssen $V$, Manner D, Kenet $G$. Venous thromboembolism in neonates and children-update 2013. Thromb Res. 2013;131:39-41.

4. Mitsunaga MM, Kogachi S, Yoon HC. Risk of Venous Thromboembolism after a Single Normal Proximal Lower Extremity Venous Ultrasound. Perm J. 2017;21:16-140.

5. Andrew M, David M, Adams M, et al. Venous thromboembolic complications (VTE) in children: first analyses of the Canadian Registry of VTE. Blood. 1994;83:1251-1257.

6. Duicu C, Bucur G, Simu I, Marginean O. Deep Venous Thrombosis Associated With Inferior Vena Cava Abnormalities And Hypoplastic Kidney In Siblings. Acta Medica Marisiensis. 2016;62:266-268.

7. Malec L, Young $\mathrm{G}$. Treatment of Venous Thromboembolism in Pediatric Patients. Front Pediatr. 2017;5:26.

8. Bizzaro MJ, Dembry LM, Baltimore RS, Gallagher PG. Casecontrol analysis of endemic Serratia marcescens bacteremia in a neonatal intensive care unit. Arch Dis Child Fetal Neonatal Ed. 2007;92:120-126.

9. Meremikwu MM, Nwachukwu CE, Asuquo AE, Okebe JU, Utsalo SJ. Bacterial isolates from blood cultures of children with suspected septicemia in Calabar, Nigeria. BMC Infect Dis. 2005;5:110-115.

10. Negussie A, Mulugeta G, Bedru A, et al. Bacteriological Profile and Antimicrobial Susceptibility Pattern of Blood Culture Isolates among Septicemia Suspected Children in Selected Hospitals Addis Ababa, Ethiopia. Int J Biol Med Res. 2015;6:4709-4717.

11. Meliț LE, Mărginean CO, Georgescu A, Duicu C. Complications of sepsis in infant. A case report. J Crit Care Med. 2016;2:9699.

12. Scarlatescu E, Tomescu D, Arama SS. Sepsis-Associated Coagulopathy. J Crit Care Med. 2016;2:156-163.

13. Setty BA, O'Brien SH, Kerlin BA. Pediatric venous thromboembolism in the United States: a tertiary care complication of chronic diseases. Pediatr Blood Cancer. 2012;59:258-264.

14. Van Ommen $\mathrm{CH}$, Heijboer $\mathrm{H}$, Büller HR, Hirasing RA, Heijmans HS, Peters M. Venous thromboembolism in childhood: a prospective two-year registry in The Netherlands. J Pediatr. 2001;139:676-681.

15. Naess IA, Christiansen SC, Romundstad P, Cannegieter SC, Rosendaal FR, Hammerstrøm J. Incidence and mortality of venous thrombosis: a population-based study. J Thromb Haemost. 2007;5:692-699.

16. Rühle $F$, Stoll $M$. Advances in predicting venous thromboembolism risk in children. Br J Haematol. 2017 Dec 19. doi: 10.1111/bjh.15060. [Epub ahead of print]

17. Van Ommen $\mathrm{CH}$, Heijboer $\mathrm{H}$, van den Dool EJ, Hutten BA, Peters M. Pediatric venous thromboembolic disease in one single center: congenital prothrombotic disorders and the clinical outcome. J Thromb Haemost. 2003;1:2516-2522.

18. Spentzouris G, Scriven RJ, Lee TK, Labropoulos N. Pediatric venous thromboembolism in relation to adults. J Vasc Surg. 2012;55:1785-1793.

19. Young $\mathrm{G}$, Albisetti $\mathrm{M}$, Bonduel $\mathrm{M}$, et al. Impact of inherited thrombophilia on venous thromboembolism in children: a systematic review and meta-analysis of observational studies. Circulation. 2008;118:1373-1382. 
Available online at: www.jccm.ro

20. Tuckuviene R, Christensen AL, Helgestad J, Johnsen SP, Kristensen SR. Pediatric venous and arterial noncerebral thromboembolism in Denmark: a nationwide populationbased study. J Pediatr. 2011;159:663-669.

21. Makhoul IR, Sujov P, Smolkin T, et al. Pathogen-specific early
The Journal of Critical Care Medicine 2018;4(1) • 33

mortality in very low birth weight infants with late-onset sepsis: a national survey. Clin Infect Dis. 2005;40:218-224.

22. Benjamin DK, DeLong ER, Cotton CM, et al. Postconception age and other risk factors associated with mortality following gram-negative rod bacteremia. J Perinatol. 2004;24:169-174. 